\title{
Antidiabetic Drugs and Their Nanoconjugates Repurposed as Novel Antimicrobial Agents against Acanthamoeba castellanii
}

\author{
Ayaz Anwar ${ }^{1 *}$, Ruqaiyyah Siddiqui ${ }^{1}$, Muhammad Raza Shah ${ }^{2}$, and Naveed Ahmed Khan ${ }^{1}$ \\ ${ }^{1}$ Department of Biological Sciences, School of Science and Technology, Sunway University, Selangor, 47500, Malaysia \\ ${ }^{2}$ HEJ Research Institute of Chemistry, International Center for Chemical and Biological Sciences, University of Karachi, Pakistan
}

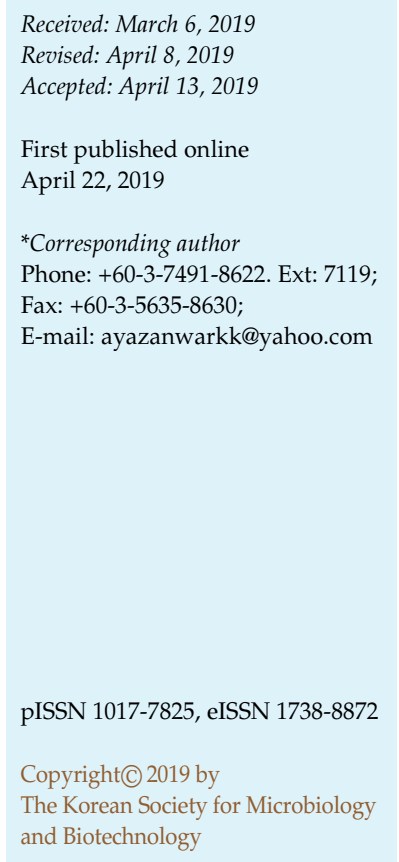

E-mail: ayazanwarkk@yahoo.com

\section{Introduction}

Acanthamoeba spp. have a worldwide distribution and are the most common ameoba found in the environment [1]. Acanthamoeba spp. cause a frequent eye infection called Acanthamoeba keratitis, and a brain infection known as granulomatous amoebic encephalitis (GAE) [2]. Contact lens wearers are more prone to get Acanthamoeba keratitis [3]. In contrast, GAE most commonly affects immunocompromised patients [4]. The life cycle of Acanthamoeba consists of two forms; the active trophozoite, and a doublewall protected, stress-resistant cyst [2]. The first line of treatment against Acanthamoeba keratitis includes 0.02\% polyhexamethylene biguanide or chlorhexidine along with $0.1 \%$ propamidine or hexamidine [5]. The treatment is unspecific and usually has limited effectiveness against the cyst stage [6]. The use of combination therapy is of value in the early stages of infections but drug resistance, side effects and toxicity have been observed [7]. Various repurposing of drugs has also shown potential against Acanthamoeba such as antineoplastic, antifungal, antibiotic, antiseptic, calcium channel blockers, antiarrhythmics, and antiparkinsonian drugs [8].

Nanoparticles have shown tremendous utility against infectious diseases [9]. However, to date there are few reports of nanoparticle applications against Acanthamoeba. Borase at al., showed the amoebicidal activity of photosynthesized silver nanoparticles [10]. In another report, titanium oxide nanoparticles are shown to reduce the viability of Acanthamoeba via photochemotherapy [11]. Silver nanoparticles have also been used to inhibit microbial growth and colonization on contact lenses, which shows the usefulness of nanoparticles in the development of safer contact lens solutions against Acanthamoeba [12]. In 
Table 1. Structures and molecular masses of Glimeperide, Vildagliptin, and Repaglinide.

\begin{tabular}{|c|c|c|}
\hline Drugs & $\begin{array}{l}\text { Molecular } \\
\text { mass }\end{array}$ & Structure \\
\hline Glimepiride & 490.617 & \\
\hline Vildagliptin & 303.399 & \\
\hline Repaglinide & 452.586 & \\
\hline
\end{tabular}

our recent studies, nanoparticles conjugated with drugs were shown to enhance the antiacanthamoebic effects of chlorhexidine, amphotericin B, nystatin and fluconazole against Acanthamoeba [13, 14].

In a recent review, we originated the idea of using carbohydrate-targeting drugs against Acanthamoeba spp. specifically to target the cyst walls [15], based on previous determinations of the walls' carbohydrate components [16]. Glycogen phosphorylase and cellulase have been previously identified as efficient targets to degrade the essential glycosidic linkages of the targeted cysts [17, 18]. Among these carbohydrate-targeting agents, anti diabetic drugs are of prime interest. Previously, the antidiabetic drug metformin (biguanide) has shown some limited effects against Acanthamoeba [1]. For the first time, we herein report the effects of 3 antidiabetic drugs (Table 1) including Glimepiride (sulfonyl urea), Vildagliptin (dipeptidyl peptidase- 4 inhibitor), and Repaglinide (meglitinides such as glucuronidation agents) against a keratitis isolate of A. castellanii belonging to the T4 genotype. All three drugs tested are FDA approved and clinically available, which may provide a head start in their drug development against Acanthamoeba keratitis. Additionally, we successfully achieved the conjugation of silver nanoparticles with vildagliptin which resulted in enhanced antiacanthamoebic activity. Amoebicidal, encystation, and excystation assays were carried out to study the antiacanthamoebic effects of the three antidiabetic drugs. Furthermore, their host cell cytotoxicity was also evaluated by lactate dehydrogenase determination.

\section{Materials and Methods}

\section{Materials}

All reagents, drugs and materials were purchased from SigmaAldrich (analytical grade) unless stated otherwise.

\section{A. castellanii Cultures}

A clinical isolate of A. castellanii (ATCC 50492) belonging to the T4 genotype, was cultured as described previously [19], in $10 \mathrm{ml}$ PYG medium \{proteose peptone $(0.75 \% \mathrm{w} / \mathrm{v})$, yeast extract $(0.75 \%$ $\mathrm{w} / \mathrm{v})$, and glucose $(1.5 \% \mathrm{w} / \mathrm{v})\}$ at $30^{\circ} \mathrm{C}$ in $75-\mathrm{cm}^{2}$ tissue culture flasks. For amoebicidal and encystation assays, A. castellanii trophozoites that adhere to the flask surface were used. These active trophozoites were detached by changing the media with phosphate buffer saline (PBS) and leaving the culture flask on ice for $15 \mathrm{~min}$ followed by gentle tapping for roughly $5 \mathrm{~min}$. Finally, the $A$. castellanii trophozoite suspension was transferred to a $50-\mathrm{ml}$ centrifuge tube, and centrifugated at 2,500 $\times g$ for $10 \mathrm{~min}$ to get the amoeba pellet. The A. castellanii pellet was resuspended in $1 \mathrm{ml}$ PBS, counted using a hemocytometer and used for amoebicidal and encystation assays.

\section{Henrietta Lacks Cervical Adenocarcinoma Cells (HeLa) Cells Culture}

HeLa cells were routinely cultured in $75-\mathrm{cm}^{2}$ culture flasks in Roswell Park Memorial Institute (RPMI)-1640 supplemented with $10 \%$ fetal bovine serum (FBS), 10\% Nu-serum, $2 \mathrm{mM}$ glutamine, $1 \mathrm{mM}$ pyruvate, penicillin (100 units $/ \mathrm{ml})$, streptomycin $(100 \mu \mathrm{g} / \mathrm{ml})$, non-essential amino acids, and vitamins. $2 \mathrm{ml}$ trypsin was added after removal of old media to obtain the cell suspension which was centrifuged for $5 \mathrm{~min}$ at $2,000 \times g$. The supernatant was aspirated and the cell pellet was resuspended in $30 \mathrm{ml}$ fresh cell growth media. $200 \mu \mathrm{l}$ of cell suspension was seeded per well in a 96-well plate and the plate was incubated at $37^{\circ} \mathrm{C}$ in a $5 \% \mathrm{CO}_{2}$ incubator with $95 \%$ humidity for at least $24 \mathrm{~h}$. The plates were observed under an inverted light microscope until formation of a uniform monolayer of HeLa cells and used for cytotoxicity assays.

\section{Amoebicidal Assay}

Amoebicidal activity of drugs was determined by incubating $5.0 \times 10^{5}$ A. castellanii trophozoites per well with various concentrations over a period of $24 \mathrm{~h}$ at $30^{\circ} \mathrm{C}$ in PBS [20]. Amoebae in PBS and treated with Chlorhexidine were considered as negative and positive controls. After incubation, A. castellanii viability was estimated by adding 0.1\% trypan blue and enumerating the numbers of live (non-stained) amoeba using a hemocytometer.

\section{Encystation Assay}

For encystation assays, $5.0 \times 10^{5} \mathrm{~A}$. castellanii trophozoites were incubated with 100 and $50 \mu \mathrm{M}$ concentrations of drugs and nanoconjugates in 1.5-ml centrifuge tubes at room temperature for $10 \mathrm{~min}$. A 24-well plate was seeded with encystation medium containing $50 \mathrm{mM} \mathrm{MgCl}_{2}$ and $10 \%$ glucose, and test samples and 
controls treated with $A$. castellanii were added in the above 24-well plates. The cells were incubated at $30^{\circ} \mathrm{C}$ for $72-96 \mathrm{~h}$ [16]. Following this incubation, $0.5 \%$ sodium dodecyl sulfate (SDS) was added in each well and left for $10 \mathrm{~min}$ to solubilize trophozoites and immature cysts while only the SDS resistant cysts were enumerated using a hemocytometer.

\section{Excystation Assay}

A. castellani $\left(1.0 \times 10^{6}\right)$ active trophozoites were harvested on nonnutrient agar plates $(1.5 \%$ bacteriological agar dissolved in water, followed by autoclaving and then spreading on petri plates), which were incubated for 10-14 days. After routine observation for cyst formation under light microscope, cysts were scraped using a cell scraper with PBS, enumerated, and stored in PBS at $4^{\circ} \mathrm{C}$. For excystation assay, $1.0 \times 10^{5}$ cysts were treated with 100 or $50 \mu \mathrm{M}$ concentrations of drugs, nanoconjugates, and respective controls in growth medium PYG for 72-96 h [21]. Trophozoites that emerged from the cyst stage were counted using a hemocytometer.

\section{A. castellanii Mediated Host Cell Cytotoxicity Assay}

To determine whether antidiabetic drugs and nanoconjugates reduce the cytotoxicity of $A$. castellanii against human cells, untreated and treated (100 or $50 \mu \mathrm{M}$ of drugs/drugs conjugated with AgNPs) amoebae were incubated with HeLa cells. Briefly, test samples and respective controls were incubated with A. castellanii in RPMI- 1640 for $2 \mathrm{~h}$ at $30^{\circ} \mathrm{C}$. These test samples and controls were then added to HeLa monolayers after removal of old media. Cells were incubated at $37^{\circ} \mathrm{C}$ in a $5 \% \mathrm{CO}_{2}$ incubator for $24 \mathrm{~h} \mathrm{[22].}$ After incubation, supernatants were collected from each well and cytotoxicity was determined by measuring lactate dehydrogenase (LDH) release as described previously [23]. Untreated cells were considered as negative control, whereas cells lysed with $0.1 \%$ Triton X-100 followed by 20 min incubation gave maximum LDH release which was considered as positive control. The percentages of cytotoxicity were calculated as follows: $\%$ cytotoxicity = (sample absorbance - negative control absorbance)/(positive control absorbance - negative control absorbance) $\times 100$.

\section{Synthesis of Vildagliptin-Conjugated Silver Nanoparticles}

Synthesis of Vildagliptin-conjugated silver nanoparticles (VgtAgNPs) was achieved by sodium borohydride reduction of silver nitrate [24]. Briefly, $1 \mathrm{mM}$ aqueous solution of Vildagliptin was reacted with $1 \mathrm{mM}$ silver nitrate aqueous solution in a 1:1 v/v ratio. The reaction mixture was magnetically stirred for $10 \mathrm{~min}$ before the addition of $20 \mu \mathrm{l}(4 \mathrm{mM})$ freshly prepared sodium borohydride aqueous solution. The solution was instantly changed into yellow-brown from transparent on addition of reducing agent indicating reduction of silver ions. The reaction mixture was further stirred for $2 \mathrm{~h}$ to ensure stabilization of colloids. The colloids were centrifuged at $12,000 \times g$ for $10 \mathrm{~min}$ to isolate unreacted reagents and potentially larger aggregates, and the pellet was resuspended in autoclaved deionized water. The same procedure was applied for the synthesis of Glimepiride and Repaglinide nanoconjugates, but we were unsuccessful in optimizing the formation of stable silver nanoparticles. For VgtAgNPs, the colloids were subjected to characterization by UV-Vis, FT-IR and AFM analysis for confirmation of formation, stabilization and morphology. These nanoconjugates were stored at $4^{\circ} \mathrm{C}$ for further experiments.

\section{Results}

Antidiabetic Drugs Exhibited Potent Amoebicidal Effects Amoebicidal assays revealed that the drugs Glimepiride, Vildagliptin, and Repaglinide significantly reduced the viability of $A$. castellanii (Table 2$)$ ( $p<0.05$ using two-sample $\mathrm{T}$ test and two-tailed distribution). Various concentrations of drugs were screened to observe the potent amoebicidal effects with minimal cytotoxicity. All drugs reduced the viability of $A$. castellanii to approx. $10^{4}$ number of cells as

Table 2. Antiacanthamoebic effects of Glimepiride, Vildagliptin and Repaglinide drugs alone.

\begin{tabular}{|c|c|c|c|}
\hline \multirow[b]{2}{*}{ Condition } & \multicolumn{3}{|c|}{ Number of viable $A$. castellanii (per well) } \\
\hline & $\begin{array}{c}\text { Amoebicidal assay } \\
\text { (in RPMI-1640) }\end{array}$ & $\begin{array}{c}\text { Encystation assay } \\
\text { (in encystation medium) }\end{array}$ & $\begin{array}{l}\text { Excystation assay } \\
\text { (in PYG) }\end{array}$ \\
\hline A. castellanii alone & $2.81 \times 10^{5} \pm 3.0 \times 10^{4}$ & $8.13 \times 10^{4} \pm 1.69 \times 10^{4}$ & $1.38 \times 10^{4} \pm 1.76 \times 10^{3}$ \\
\hline A. castellanii $+\mathrm{CHX}$ & $3.13 \times 10^{5} \pm 1.69 \times 10^{4}$ & $2.5 \times 10^{3} \pm 1.03 \times 10^{3}$ & $2.5 \times 10^{3} \pm 2.04 \times 10^{3}$ \\
\hline A. castellanii + Glimepiride $100 \mu \mathrm{M}$ & $4.38 \times 10^{4} \pm 1.69 \times 10^{4}$ & $1.25 \times 10^{4} \pm 1.02 \times 10^{4}$ & $2.5 \times 10^{3} \pm 2.04 \times 10^{3}$ \\
\hline A. castellanii + Glimepiride $50 \mu \mathrm{M}$ & $8.13 \times 10^{4} \pm 3.34 \times 10^{4}$ & $3.75 \times 10^{4} \pm 1.02 \times 10^{4}$ & $1.0 \times 10^{4} \pm 2.88 \times 10^{3}$ \\
\hline A. castellanii + Vildagliptin $100 \mu \mathrm{M}$ & $6.25 \times 10^{4} \pm 2.28 \times 10^{4}$ & 0 & $7.5 \times 10^{3} \pm 2.04 \times 10^{3}$ \\
\hline A. castellanii + Vildagliptin $50 \mu \mathrm{M}$ & $6.25 \times 10^{4} \pm 1.02 \times 10^{4}$ & $6.25 \times 10^{3} \pm 8.83 \times 10^{3}$ & $1.0 \times 10^{4} \pm 0$ \\
\hline A. castellanii + Repaglinide $100 \mu \mathrm{M}$ & $6.25 \times 10^{4} \pm 2.28 \times 10^{4}$ & 0 & $1.25 \times 10^{3} \pm 1.76 \times 10^{3}$ \\
\hline A. castellanii + Repaglinide $50 \mu \mathrm{M}$ & $8.13 \times 10^{4} \pm 2.22 \times 10^{4}$ & $1.25 \times 10^{4} \pm 1.02 \times 10^{4}$ & $3.75 \times 10^{3} \pm 1.76 \times 10^{3}$ \\
\hline
\end{tabular}




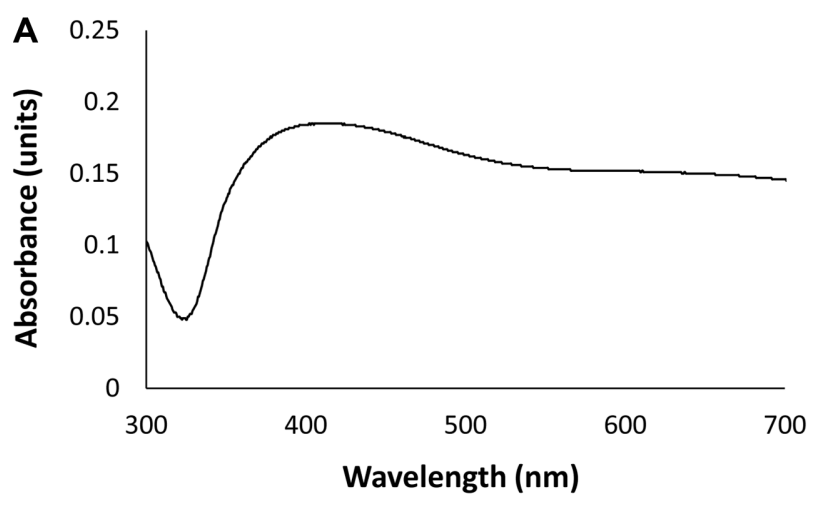

B

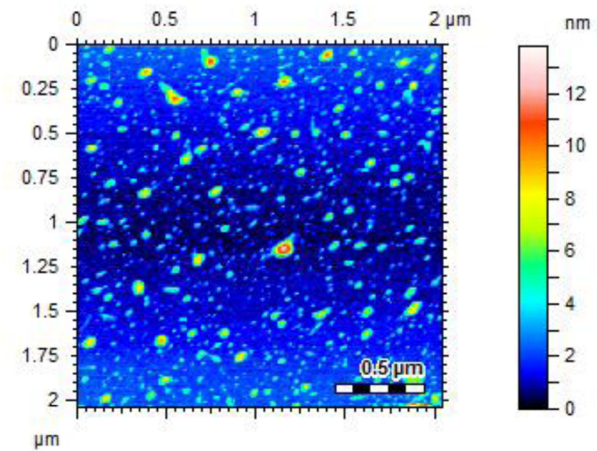

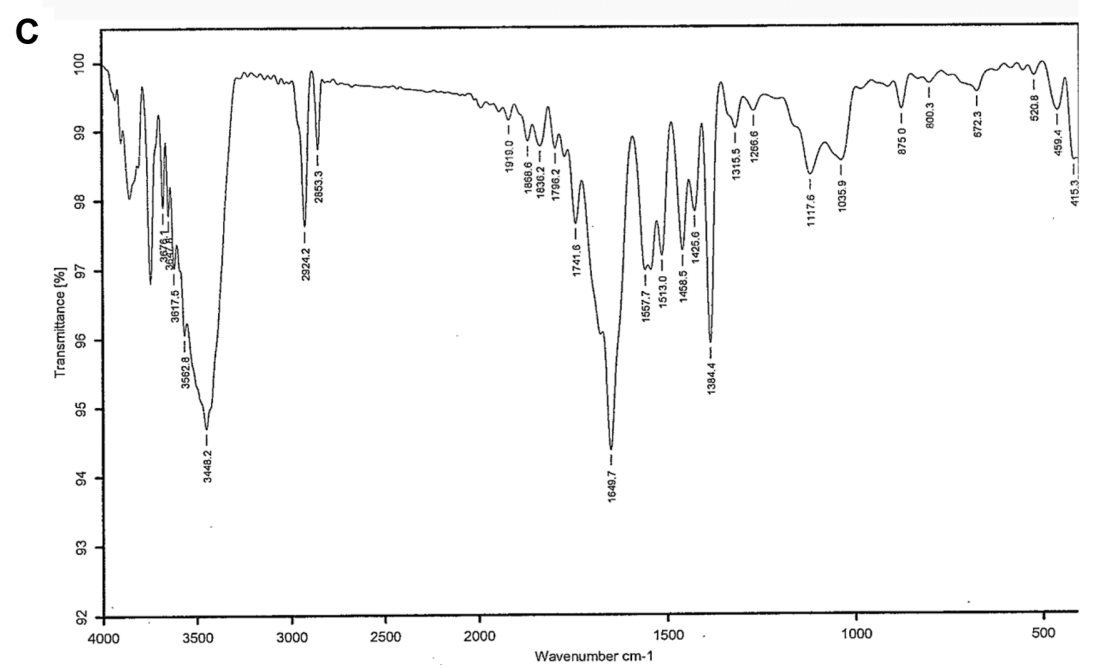

Fig. 1. (A) UV-Vis spectrum of Vgt-AgNPs. UV-Vis spectrum was recorded by UV-Vis spectrophotometer (Evolution 300, Thermo Scientific) in aqueous medium. Vgt-AgNPs showed characteristic surface plasmon resonance band at $400 \mathrm{~nm}$. (B) AFM topographic images were recorded by AFM (Agilent 5500) instrument operated in tapping mode with silicon nitride cantilever. The AFM image shows the presence of small and spherical nanoparticles of wide size distribution. The average size of Vgt-AgNPs was found to be $10 \mathrm{~nm}$. (C) FT-IR spectra of Vgt-AgNPs was obtained by using FT-IR spectrometer (Vector 22, Bruker) using Potassium bromide disc method. All characterization results presented in this figure are representatives of at least three independent experiments.

compared to relevant controls and initial inoculum i.e., $10^{5}$ at 100 and $50 \mu \mathrm{M}$ concentrations.

\section{Glimepiride, Vildagliptin and Repaglinide Inhibited Encystation and Excystation of A. castellanii}

The encystation assay revealed that all three drugs inhibited encystation of $A$. castellanii. The encystation media induced formation of cysts and $8.13 \times 10^{4}$ number of cysts were enumerated in negative control (Table 2). However, the addition of 100 and $50 \mu \mathrm{M}$ concentrations of drugs resulted in significant reduction in encystation of trophozoites $(p<0.05$ using two-sample T test and twotailed distribution). Vildagliptin and Repaglinide at $100 \mu \mathrm{M}$ caused complete blocking of encystation, and only tropho- zoites were obtained even after 96 h. Excystation effects of these antidiabetic drugs were also evaluated by treating 100 and $50 \mu \mathrm{M}$ concentrations of drugs with preformed cysts. In the favorable conditions (growth medium) A. castellanii cysts were converted into trophozoites. Two of the drugs, Glimepiride and Repaglinide, significantly reduced the excystation at $100 \mu \mathrm{M}$ (Table 2) $(p<0.05$ using two-sample $\mathrm{T}$ test and two-tailed distribution).

Characterization of Vgt-AgNPs Via UV-Visible Spectrophotometry, AFM, and FT-IR Spectroscopy

Vgt-AgNPs were characterized by UV-vis spectrophotometry, AFM microscopy, and FT-IR spectroscopy. UV-vis spectra of Vgt-AgNPs showed a characteristic surface plasmon 
Table 3. Antiacanthamoebic effects of Vildagliptin-conjugated silver nanoparticles.

\begin{tabular}{lccc}
\hline \multirow{2}{*}{ Condition } & \multicolumn{3}{c}{ Number of viable A. castellanii (per well) } \\
\cline { 2 - 4 } & $\begin{array}{c}\text { Amoebicidal assay } \\
\text { (in RPMI-1640) }\end{array}$ & $\begin{array}{c}\text { Encystation assay } \\
\text { (in encystation medium) }\end{array}$ & $\begin{array}{c}\text { Excystation assay } \\
\text { (in PYG) }\end{array}$ \\
\hline A. castellanii alone & $3.44 \times 10^{5} \pm 2.22 \times 10^{4}$ & $4.13 \times 10^{4} \pm 6.03 \times 10^{3}$ & $6.88 \times 10^{4} \pm 6.03 \times 10^{3}$ \\
A. castellanii + CHX & $2.50 \times 10^{4} \pm 1.44 \times 10^{4}$ & $2.5 \times 10^{3} \pm 1.03 \times 10^{3}$ & $8.75 \times 10^{3} \pm 1.76 \times 10^{3}$ \\
A. castellanii + AgNPs alone $10 \mu \mathrm{M}$ & $2.06 \times 10^{5} \pm 2.22 \times 10^{4}$ & $2.88 \times 10^{4} \pm 5.30 \times 10^{3}$ & $3.13 \times 10^{4} \pm 3.38 \times 10^{3}$ \\
A. castellanii + AgNPs alone $5 \mu \mathrm{M}$ & $2.56 \times 10^{5} \pm 1.69 \times 10^{4}$ & $3.50 \times 10^{4} \pm 2.88 \times 10^{3}$ & $3.75 \times 10^{4} \pm 4.56 \times 10^{3}$ \\
A. castellanii + Vildagliptin $10 \mu \mathrm{M}$ & $1.44 \times 10^{5} \pm 2.65 \times 10^{4}$ & $6.25 \times 10^{3} \pm 3.38 \times 10^{3}$ & $1.13 \times 10^{4} \pm 3.38 \times 10^{3}$ \\
A. castellanii + Vildagliptin $5 \mu \mathrm{M}$ & $2.0 \times 10^{5} \pm 1.44 \times 10^{4}$ & $8.75 \times 10^{3} \pm 1.76 \times 10^{3}$ & $2.75 \times 10^{4} \pm 4.56 \times 10^{3}$ \\
A. castellanii + Vgt-AgNPs $10 \mu \mathrm{M}$ & $3.13 \times 10^{4} \pm 1.69 \times 10^{4}$ & $5.0 \times 10^{3} \pm 2.88 \times 10^{3}$ & $1.00 \times 10^{4} \pm 2.88 \times 10^{3}$ \\
A. castellanii + Vgt-AgNPs $5 \mu \mathrm{M}$ & $6.88 \times 10^{4} \pm 8.83 \times 10^{4}$ & $6.25 \times 10^{3} \pm 3.38 \times 10^{3}$ & $1.00 \times 10^{4} \pm 2.88 \times 10^{3}$ \\
\hline
\end{tabular}

resonance (SPR) band at $400 \mathrm{~nm}$ (Fig. 1A), which suggests the formation of medium-sized silver nanoparticles. AFM analysis showed the broad size distribution and spherical morphology of the Vgt-AgNPs. The average size of the Vgt-AgNPs was found to be $10 \mathrm{~nm}$, however, due to rapid reduction by using sodium borohydride, size selectivity was neither the purpose nor achieved (Fig. 1B). The comparative FT-IR analysis of pure Vildagliptin vs VgtAgNPs shows characteristic broad peak for $\mathrm{O}-\mathrm{H}$ stretching at 3,294 $1 / \mathrm{cm}$, strong $\mathrm{C}=\mathrm{O}$ stretching at $1,6581 / \mathrm{cm}$, and weak C-N signal at 2,237 1/cm, as compared to Vgt-AgNPs which showed $\mathrm{O}-\mathrm{H}$ stretch at $3,4481 / \mathrm{cm}, \mathrm{C}=\mathrm{O}$ stretching at $1,6541 / \mathrm{cm}$ (Fig. 1C). Notably, C-N stretch peak was diminished upon formation of nanoparticles. These peak shifts and disappearance suggested the stabilization of silver nanoparticles with the hydroxyl and nitrile groups of Vildagliptin.

\section{Silver Nanoparticle Conjugation Significantly Enhanced Antiamoebic Effects of Vildagliptin}

AgNPs significantly enhanced the amoebicidal potency of Vildagliptin and exhibited pronounced cidal effects at reduced concentration of 10 and $5 \mu \mathrm{M}$ as compared to Vildagliptin and AgNPs alone (Table 3) $(p<0.05$ using two-sample $\mathrm{T}$ test and two-tailed distribution). Furthermore, Vgt-AgNPs also showed minimal encystation and excystation (Table 3). The enhanced effects of nanoparticle conjugation on Vildagliptin were clear at low concentration of $5 \mu \mathrm{M}$, while AgNPs alone had limited excystation efficacy.

\section{Antidiabetic Drugs and Nanoconjugates Reduced Host Cell Cytotoxicity Caused by A. castellanii}

$A$. castellanii invades the host cells when untreated, and to test the effects of these drugs against cytopathogenicity,
100 and $50 \mu \mathrm{M}$ of drugs were incubated for $2 \mathrm{~h}$ with A. castellanii followed by a further $24 \mathrm{~h}$-incubation with HeLa cells. Cytotoxicity was measured by using LDH determination as a measure of cell damage as described in the Materials and Methods section. A. castellanii alone produced more than $80 \%$ host cell cytotoxicity, whereas pretreatment of A.castellanii with drugs alone and nanoconjugates caused significant reduction in host cell cytotoxicity (Fig. 2A). Additionally, Vgt-AgNPs also reduced the host cell cytotoxicity of $A$. castellanii (Fig. 2B) ( $p<0.05$ using two-sample T test and two-tailed distribution).

\section{Discussion}

Currently there is no single drug effective against Acanthamoeba keratitis, however combinations of antiparasitic and broad-spectrum antimicrobials have shown a few reports of successful treatments [25]. Despite the limited activity of the drugs currently being used, the resistance and recurrence of Acanthamoeba cysts pose a major challenge in drug development. Hence, there is an urgent need to identify new therapeutic agents effective against Acanthamoeba. Recently, carbohydrate-targeting molecules and biomolecules are identified to control differentiation between cysts and trophozoites, since the cyst double walls consist of glycosides as a major contributor. In this regard, some enzymes have shown promise but their shelf life, stability and specificity in biological systems have obvious limitations as compared to drug molecules.

Glimepiride acts on ATPase-dependent potassium channels in $\beta$ cells of the pancreas to stimulate insulin release [26]. Studies investigating the molecular basis underlying the clinical profile of Glimepiride provide strong evidence for multiple molecular targets/mechanisms for the blood 

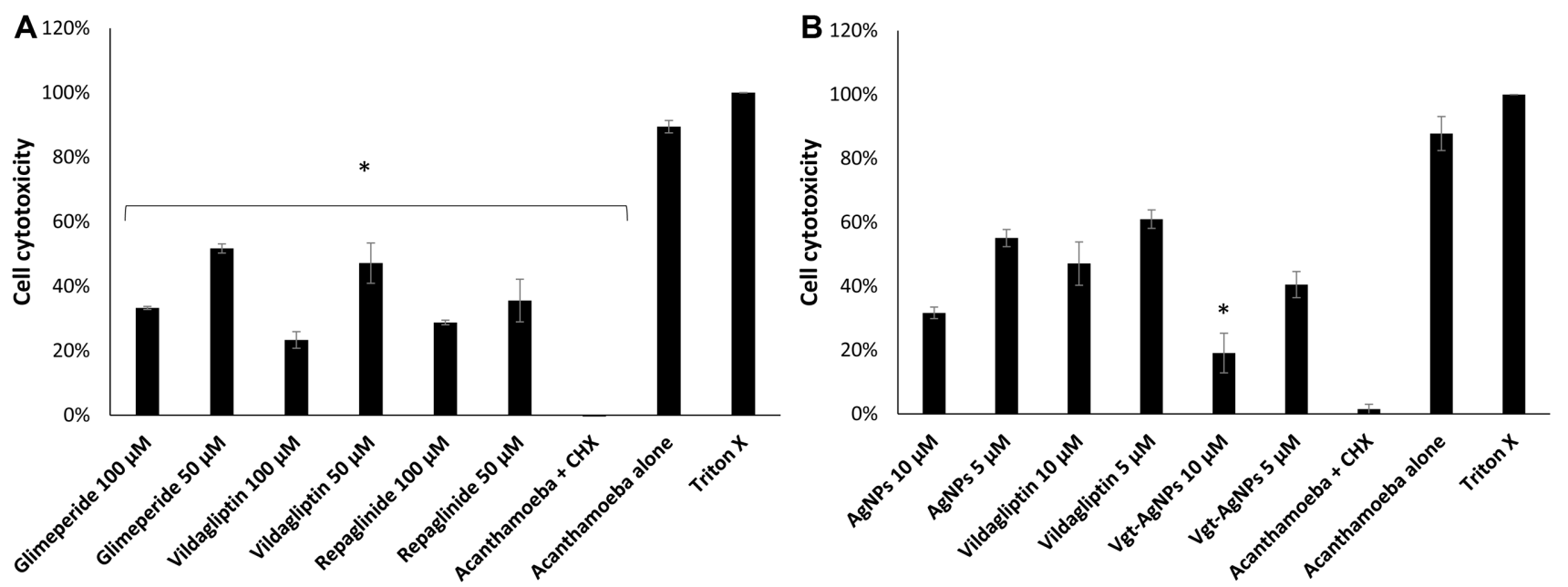

Fig. 2. (A) Pretreatment of Glimeperide, Vildagliptin, and Repaglinide reduced A. castellanii-mediated host cell cytotoxicity. The cytopathogenicity assays were performed as mentioned previously. Briefly, $1 \times 10^{5}$ amoebae were incubated at $30^{\circ} \mathrm{C}$ with antidiabetic drugs for $2 \mathrm{~h}$ in RPMI-1640 and then incubated with HeLa cells for $24 \mathrm{~h}$ at $37^{\circ} \mathrm{C}$ in a $5 \% \mathrm{CO}_{2}$ incubator. Next, cell-free supernatant was collected, and cytotoxicity was determined using Lactate dehydrogenase (LDH) assay kit. Negative control values for cytotoxicity assays were obtained by incubating HeLa cells with RPMI-1640 alone, and positive control values were obtained by $100 \%$ cell death using $0.1 \%$ Triton $\mathrm{X}-100$. The results revealed that drugs significantly reduced the host cell cytotoxicity $50 \mu \mathrm{M}$ ( $p<0.05$ using two-sample T test and two-tailed distribution). (B) Nanoconjugates reduced the pathogenicity more effectively as compared to Vildaglitin and AgNPs alone.

glucose-lowering effect of Glimepiride operating on both pancreatic B-cells and extrapancreatic cells [27]. Abd ElWahed et al., synthesized several new lanthanide Glimepiride complexes which showed potent antimicrobial activity against bacteria and fungi [28]. Vildagliptin is an adamantly small molecule that by inhibition of dipeptidyl peptidase- 4 (DPP4) prevents degradation of glucagon-like peptide-1 (GLP-1) which may suggest that it affects saccharides/ metabolic functions in amoeba. It binds covalently to the catalytic site of DPP-4, eliciting prolonged enzyme inhibition. Vildagliptin has been shown to stimulate insulin secretion and inhibit glucagon secretion in a glucose-dependent manner [29]. Vildagliptin also inhibits glucose production, as determined with a variety of methods. Al-Abdullah et al., reported the synthesis, antimicrobial and hypoglycemic activities of novel $\mathrm{N}$-(1-Adamantyl) carbothioamide derivatives related to the gliptin class of drugs [30]. More recently, Vildagliptin has been used to synthesize nanomaterials for physical and biological applications [31, 32]. Repaglinide is a new carbamoylmethyl benzoic acid derivative that is structurally related to meglitinide. Like all other active oral hypoglycaemic agents, it displays a comparable U-shaped configuration. Repaglinide exerts its effects by binding to a site on the plasma membrane of $\beta$ - cells, thereby closing ATP-sensitive potassium channels. This causes depolarization of the $\beta$-cell and the opening of voltage-sensitive calcium channels allowing the influx of extracellular calcium ions. This increase in intracellular calcium, in turn, stimulates insulin release [33]. Previous studies have suggested that changes in flux of calcium can lead to amoebae cell damage [34, 35], which may explain findings observed in this study.

Nanoparticles provide stable platforms for the targeted drug delivery [36]. More recently, stimuli responsive nanoconjugates have shown incredible applicability against bacteria and other pathogens [37]. Moreover, nanoparticles are anticipated as next-generation antimicrobial agents due to their desired and tunable applications in chemotherapy of infectious diseases [9]. Among various types of nanoparticles, silver nanoparticles are one of the most abundant materials used in biomedicine research. The known chemistry, bio-inertness, stability, and interactions with DNA and specific biomolecules of silver nanoparticles make them an ideal candidate for antimicrobial applications [37]. Silver nanoparticles like any other nanoparticles are known to increase the bioavailability of loaded drugs due to their small size and high drug-loading capability etc. However, Vildagliptin-conjugated nanoparticles have been 
tested against Acanthamoeba castellanii for the first time. Previous study [24] has shown that these nanoparticles exhibit antibacterial effects by production of reactive oxygen species. Hence, it is suggested that the enhanced amoebicidal effects of Vildagliptin nanoconjugates as compared to the drug alone have been observed because of the synergistic effects with the production of reactive oxygen species along with metabolic inhibition of amoeba.

In conclusion, Glimepiride, Vildagliptin, and Repaglinide alone and Vgt-AgNPs were shown to exhibit antiacanthamoebic potency against both trophozoite and cyst forms of Acanthamoeba castellanii for the first time. In addition, silver nanoparticle conjugation improved the overall activity of Vildagliptin against $A$. castellanii at much reduced concentration. Hence, these antidiabetic drugs may serve as potential targets in the treatment and management of A. castellanii infections. The most effective application of these nanoparticles in our opinion would be topical administration in the eye against Acanthamoeba keratitis since it can withstand a relatively higher concentration as compared to oral or any other mode of administration. Our future interests are to develop the in vivo applicability of these drugs and nanoconjugates, as well as exploring other carbohydrates targeting compounds.

\section{Acknowledgments}

This work is supported by the University Research Award, Sunway University, Malaysia.

\section{Conflict of Interest}

The authors have no financial conflicts of interest to declare.

\section{References}

1. Seal DV. 2003. Acanthamoeba keratitis update-incidence, molecular epidemiology and new drugs for treatment. Eye 17: 893-905.

2. Marciano-Cabral F, Cabral G. 2003. Acanthamoeba spp. as agents of disease in humans. Clin. Microbiol. Rev. 16: 273-307.

3. Illingworth CD, Cook SD, Karabatsas CH, Easty DL. 1995. Acanthamoeba keratitis: risk factors and outcome. Br. J. Ophthalmol. 79: 1078-1082.

4. Visvesvara GS, Moura H, Schuster FL. 2007. Pathogenic and opportunistic free-living amoebae: Acanthamoeba spp., Balamuthia mandrillaris, Naegleria fowleri, and Sappinia diploidea. FEMS Immun. Med. Microbiol. 50: 1-26.
5. Khan NA. 2006. Acanthamoeba: biology and increasing importance in human health. FEMS Microbiol. Rev. 30: 564-595.

6. Lorenzo-Morales J, Khan NA, Walochnik J. 2015. An update on Acanthamoeba keratitis: diagnosis, pathogenesis and treatment. Parasite 2015: 22: 10.

7. Coulon C, Collignon A, McDonnell G, Thomas V. 2010. Resistance of Acanthamoeba cysts to disinfection treatments used in health care settings. J. Clin. Microbiol. 48: 2689-2697.

8. Ortillés Á, Belloc J, Rubio E, Fernández MT, Benito M, Cristóbal JÁ, et al. 2017. In-vitro development of an effective treatment for Acanthamoeba keratitis. Int. J. Antimicrob. Agents 50: 325-333.

9. Huh AJ, Kwon YJ. 2011. "Nanoantibiotics": a new paradigm for treating infectious diseases using nanomaterials in the antibiotics resistant era. J. Control. Release 156: 128-145.

10. Borase HP, Patil CD, Sauter IP, Rott MB, Patil SV. 2013. Amoebicidal activity of phytosynthesized silver nanoparticles and their in vitro cytotoxicity to human cells. FEMS Microbiol. Lett. 345: 127-131.

11. Imran M, Muazzam AG, Habib A, Matin A. 2016. Synthesis, characterization and amoebicidal potential of locally synthesized $\mathrm{TiO}_{2}$ nanoparticles against pathogenic Acanthamoeba trophozoites in vitro. J. Photochem. Photobiol. B: Biol. 159: 125-132.

12. Willcox MD, Hume EB, Vijay AK, Petcavich R. 2010. Ability of silver-impregnated contact lenses to control microbial growth and colonisation. J. Optometry 3: 143-148.

13. Aqeel Y, Siddiqui R, Anwar A, Shah MR, Khan NA. 2016. Gold nanoparticle conjugation enhances the antiacanthamoebic effects of chlorhexidine. Antimicrob. Agents Chemother. 60: 1283-1288.

14. Anwar A, Siddiqui R, Shah MR, Khan NA. 2019. Gold nanoparticles conjugation enhances antiacanthamoebic properties of nystatin, fluconazole and amphotericin B. J. Microbiol. Biotechnol. 29: 171-177.

15. Anwar A, Khan NA, Siddiqui R, 2018. Combating Acanthamoeba spp. cysts: what are the options? Parasit. Vectors 11: 26.

16. Dudley R, Jarroll EL, Khan NA. 2009. Carbohydrate analysis of Acanthamoeba castellanii. Exp. Parasitol. 122: 338-343.

17. Lorenzo-Morales J, Kliescikova J, Martinez-Carretero E, De Pablos LM, Profotova B, Nohynkova E, et al. 2008. Glycogen phosphorylase in Acanthamoeba spp.: determining the role of the enzyme during the encystment process using RNA interference. Eukaryot. Cell. 7: 509-517.

18. Abjani F, Khan NA, Yousuf FA, Siddiqui R. 2016. Targeting cyst wall is an effective strategy in improving the efficacy of marketed contact lens disinfecting solutions against Acanthamoeba castellanii cysts. Cont. Lens Anterior Eye 39: 239243.

19. Sissons J, Alsam S, Stins M, Rivas AO, Morales JL, Faull J, et al. 2006. Use of in vitro assays to determine effects of human serum on biological characteristics of Acanthamoeba castellanii. J. Clin. Microbiol. 44: 2595-2600. 
20. Anwar A, Siddiqui R, Hussain MA, Ahmed D, Shah MR, Khan NA. 2018. Silver nanoparticle conjugation affects antiacanthamoebic activities of amphotericin B, nystatin, and fluconazole. Parasitol. Res. 117: 265-271.

21. Lakhundi S, Khan NA, Siddiqui R. 2014. Inefficacy of marketed contact lens disinfection solutions against keratitis-causing Acanthamoeba castellanii belonging to the T4 genotype. Exp. Parasitol. 141: 122-128.

22. Sissons J, Kim KS, Stins M, Jayasekera S, Alsam S, Khan NA. 2005. Acanthamoeba castellanii induces host cell death via a phosphatidylinositol 3-kinase-dependent mechanism. Infect. Immun. 73: 2704-2708.

23. Anwar A, Siddiqui R, Shah MR, Khan NA. 2018. Gold nanoparticle-conjugated cinnamic acid exhibits antiacanthamoebic and antibacterial properties. Antimicrob. Agents Chemother. 62: e00630-18.

24. Masri A, Anwar A, Ahmed D, Siddiqui R, Shah MR, Khan N. 2018. Silver nanoparticle conjugation enhanced antibacterial efficacy of clinically approved drugs Cephradine and Vildagliptin. Antibiotics 7: 100.

25. Debnath A, Tunac JB, Silva-Olivares A, Galindo-Gómez S, Shibayama M, McKerrow JH. 2014. In vitro efficacy of corifungin against Acanthamoeba castellanii trophozoites and cysts. Antimicrob. Agents Chemother. 58: 1523-1528.

26. Campbell RK. 1998. Glimepiride: role of a new sulfonylurea in the treatment of type 2 diabetes mellitus. Ann. Pharmacother. 32: 1044-1052.

27. Muller G. 2005. The mode of action of the antidiabetic drug glimepiride-beyond insulin secretion. Immunol. Endocr. Metab. Agents Med. Chem. 5: 499-518.

28. Abd El-Wahed M, El-Megharbel S, El-Sayed M, Zahran Y, Refat M. 2013. Synthesis of several new lanthanide Glimepiride complexes for evaluation of microbial activity. Russ. J. Gen. Chem. 83: 2438-2446.

29. Ahrén B, Schweizer A, Dejager S, Villhauer EB, Dunning BE, Foley JE. 2011. Mechanisms of action of the dipeptidyl peptidase-4 inhibitor vildagliptin in humans. Diabetes Obes. Metab. 13: 775-783.

30. Al-Abdullah E, Al-Tuwaijri H, Hassan H, Al-Alshaikh M, Habib E, El-Emam A. 2015. Synthesis, antimicrobial and hypoglycemic activities of novel N-(1-adamantyl) carbothioamide derivatives. Molecules 20: 8125-8143.

31. Waghulde M, Naik J. 2017. Comparative study of encapsulated vildagliptin microparticles produced by spray drying and solvent evaporation technique. Drying Technol. 35: 1644-1654.

32. Baig MMFA, Khan S, Naeem MA, Khan GJ, Ansari MT. 2018. Vildagliptin loaded triangular DNA nanospheres coated with eudragit for oral delivery and better glycemic control in type 2 diabetes mellitus. Biomed. Pharmacother. 97: 1250-1258.

33. Malaisse WJ. 1999. Repaglinide, a new oral antidiabetic agent: a review of recent preclinical studies. Eur. J. Clin. Invest. 29: 21-29.

34. Baig AM, Iqbal J, Khan NA. 2013. In vitro efficacies of clinically available drugs against growth and viability of an Acanthamoeba castellanii keratitis isolate belonging to the T4 genotype. Antimicrob. Agents Chemother. 57: 3561-3567.

35. Baig AM, Zuberi H, Khan NA. 2014. Recommendations for the management of Acanthamoeba keratitis. J. Med. Microbiol. 63: 770-771.

36. Kumari A, Yadav SK, Yadav SC. 2010. Biodegradable polymeric nanoparticles based drug delivery systems. Colloids Surf. B: Biointerfaces 75: 1-8.

37. De las Heras Alarcón C, Pennadam S, Alexander C. 2005. Stimuli responsive polymers for biomedical applications. Chem. Soc. Rev. 34: 276-285.

38. Sondi I, Salopek-Sondi B. 2004. Silver nanoparticles as antimicrobial agent: a case study on E. coli as a model for Gram-negative bacteria. J. Colloids Interface Sci. 275: 177-182. 